\title{
AUTOMATIC REDUCTION OF ARTIFACTS IN EEG-SIGNALS
}

\author{
Daniela Schachinger ${ }^{1}$, Kaspar Schindler ${ }^{2}$ and Tilmann Kluge ${ }^{1}$ \\ ${ }^{1}$ Austrian Research Centers GmbH - ARC; Donau-City-Str. 1, 1220 Vienna, Austria \\ ${ }^{2}$ Inselspital - Department for Neurology, 3010 Bern, Switzerland
}

\begin{abstract}
Electroencephalograms (EEG) are often contaminated with high amplitude artifacts limiting the usability of data. Methods that reduce these artifacts are often restricted to certain types of artifacts, require manual interaction or large training data sets. Within this paper we introduce a novel method, which is able to eliminate many different types of artifacts without manual intervention. The algorithm first decomposes the signal into different sub-band signals in order to isolate different types of artifacts into specific frequency bands. After signal decomposition with principal component analysis (PCA) an adaptive threshold is applied to eliminate components with high variance corresponding to the dominant artifact activity. Our results show that the algorithm is able to significantly reduce artifacts while preserving the EEG activity. Parameters for the algorithm do not have to be identified for every patient individually making the method a good candidate for preprocessing in automatic seizure detection and prediction algorithms.
\end{abstract}

Index Terms - artifacts, EEG, PCA

\section{INTRODUCTION}

EEG-signals are often contaminated by undesired dominant activity with very high amplitude relative to the amplitude of the desired signal. Such artifacts often cover the activity of interest and limit the usability of data. The different types of artifacts can have physiological origin, e.g. muscle contractions, body motion or eye blinks, or can be due to technical reasons like loose electrodes or cable movement.

Several methods exist that can detect artifacts and the affected data can be rejected in further processing [1, 2, 3]. However, it is often not appropriate to disregard the signal within these regions. A prominent example where these data must be preserved are EEGsignals for the automatic diagnosis of epilepsy. In these long-time recordings lasting several days, signals from the ictal periods are often covered by artifacts caused by convulsions and other clinical signs of the seizure.

Therefore methods that reduce artifacts in the signals leaving the activity of interest mostly unchanged have been developed. Most of these methods decompose the signals such that the activity of interest and artifact activity are separated into different components. Common decomposition techniques in this context are independent component analysis (ICA), wavelet decomposition or PCA. Components containing mainly artifact activity get eliminated and the signal is reconstructed from the remaining components. As a major disadvantage for automatic signal processing, some of these methods require a trained supervisor who visually detects the relevant components $[4,5]$. In contrast, algorithms that can detect artifacts automatically without supervision, e.g. by correlating components with an average signal of the artifact, are often limited to a specific type of artifact $[6,7,8,9]$.
Another method exists that simultaneously reduces different kinds of artifacts automatically by applying a Bayesian classifier to signals decomposed by ICA and determine the probability of representing EEG-activity [10]. However, this method requires a large amount of supervised data for parameter identification prior to online processing.

In this paper we present a novel approach for automatic reduction of artifacts without sacrificing the EEG-activity. This algorithm first decomposes the signal into different sub-bands in order to isolate different types of artifacts into specific frequency bands. After signal decomposition using PCA a signal adaptive threshold is applied to eliminate components with high variance.

\section{METHOD}

\subsection{Algorithm}

The method proposed in this paper works on a set of simultaneously recorded signals $S_{m}[n], m=1,2, \ldots, M, n \in \mathbb{Z}$, which are obtained from $M$ different recording sites. Using a suitable filterbank, each signal is decomposed into $I$ sub-band signals $S_{m, i}[n], i=$ $1,2, \ldots, I$.

Within a moving window of length $N$ and overlap $L$ the signal is written in vector-notation

$$
\begin{gathered}
\mathbf{d}_{m, i}^{(w)}=\left[S_{m, i}[w(N-L)], S_{m, i}[w(N-L)+1], \ldots,\right. \\
\left.S_{m, i}[w(N-L)+N-1]\right]^{T} .
\end{gathered}
$$

The index $w$ indicates the temporal position of the window. These vectors are the columns of the $N \times M$ matrices

$$
D_{i, w}=\left[\mathbf{d}_{1, i}^{(w)}, \mathbf{d}_{2, i}^{(w)}, \ldots, \mathbf{d}_{M, i}^{(w)}\right]
$$

that completely represent the $i$-th sub-band of the $w$-th window.

For the PCA of the matrices $D_{i, w}$, the singular value decomposition (SVD) of $D_{i, w}$ is used:

$$
D_{i, w}=U_{i, w} \Sigma_{i, w} V_{i, w}^{T} .
$$

$U_{i, w} \in \mathbb{R}^{N \times N}$ and $V_{i, w} \in \mathbb{R}^{M \times M}$ are orthonormal transformation matrices, respectively. The matrix $\Sigma_{i, w} \in \mathbb{R}^{N \times M}$ is a diagonal matrix with monotonically decreasing diagonal entries $\sigma_{1}^{(i, w)}, \sigma_{2}^{(i, w)}, \ldots, \sigma_{M}^{(i, w)}$, which are the singular values of $D_{i, w}$. The principal components are the columns of

$$
P_{i, w}=D_{i, w} V_{i, w},
$$

which can be rewritten as (cf. (3))

$$
P_{i, w}=U_{i, w} \Sigma_{i, w} V_{i, w}^{T} V_{i, w}=U_{i, w} \Sigma_{i, w} .
$$

Since the orthonormal columns of $U_{i, w}$ define the directions of the principal components, it can be seen from (4) that the singular values 
in $\Sigma_{i, w}$ can be interpreted as an estimator for the variances of the principal components.

A fundamental feature of our method is the adaptive thresholding scheme for the elimination of undesired components. This scheme is based on a measure $\bar{\Theta}_{i, w}$ that is defined as a function of the singular values $\left\{\sigma_{m}^{(i, w)} \mid m=1,2, \ldots, M\right\}$. Suitable functions here are, e.g., the $k$-percentile $\mathcal{P}_{k}$ or the mean $\overline{\sigma_{m}}$ of the singular values. Using $\bar{\Theta}_{i, w}$ the signal adaptive threshold with values $\Theta_{i, w}$ can be defined. An intermediate threshold $\tilde{\Theta}_{i, w}$ can be adjusted stepwise by

$$
\tilde{\Theta}_{i, w}=\left(1-\alpha_{i}\right) \Theta_{i, w-1}+\alpha_{i} \bar{\Theta}_{i, w}
$$

with $\alpha_{i} \in[0,1]$. For large ratios between the preceding and the intermediate threshold value, $\Theta_{i, w-1}$ and $\tilde{\Theta}_{i, w}$, respectively, the threshold does not get adapted yielding in the condition

$$
\Theta_{i, w}=\left\{\begin{array}{ll}
\tilde{\Theta}_{i, w} & , \text { if } \tilde{\Theta}_{i, w}<\beta_{i} \Theta_{i, w-1} \\
\Theta_{i, w-1} & , \text { else }
\end{array},\right.
$$

with $\beta_{i}>1$. Note that $\alpha_{i}$ defines the degree of adaption and $\beta_{i}$ controls the maximum step size between two succeeding threshold values. Hence the smaller the values of $\alpha_{i}$ and $\beta_{i}$ are chosen, the smoother is the course of the adaptive threshold.

In order to eliminate components in $P_{i, w}$ an $M \times M$ diagonal matrix $T_{i, w}$ is defined with entries $t_{1}^{(i, w)}, t_{2}^{(i, w)}, \ldots, t_{M}^{(i, w)}$ in the diagonal. The values $t_{m}^{(i, w)}$ are obtained by comparison of the singular values with a scaled version of $\Theta_{i, w}$ with $\gamma_{i}>1$ according to

$$
t_{m}^{(i, w)}= \begin{cases}1, & \text { if } \sigma_{m}^{(i, w)}<\gamma_{i} \Theta_{i, w} . \\ 0, & \text { else }\end{cases}
$$

Multiplication of the principal component matrix $P_{i, w}$ with $T_{i, w}$ results in the modified matrix

$$
\tilde{P}_{i, w}=P_{i, w} T_{i, w} .
$$

In this matrix $\tilde{P}_{i, w}$ the principal components above threshold are simply set to zero.

Singular values can show very small fluctuations over a long period of time. If the adaptive threshold $\Theta_{i, w}$ is within the range of these fluctuations, corresponding principal components can be eliminated in one window but retained in the succeeding. This can lead to spurious fluctuations of the signal after automatic artifact reduction. To avoid this effect, $T_{i, w}$ is modified such that successive components are either continuously eliminated or retained, as long as two consecutive singular values do not differ by more than $3 \%$.

The next step is a back-transformation into the original signal space by (cf. (3))

$$
\hat{D}_{i, w}=\tilde{P}_{i, w} V_{i, w}^{T},
$$

and multiplication with an appropriate window matrix $F_{i, w}$ as

$$
\tilde{D}_{i, w}=F_{i, w} \hat{D}_{i, w} .
$$

This window matrix is an $N \times N$ diagonal matrix with entries $f_{i, w}[\nu], \nu=1,2, \ldots, N . f_{i, w}[\nu]$ is a window function with an appropriate roll-off in the region of overlapping samples. This avoids discontinuities when components are cancelled and distorted amplitudes due to the following overlap-add procedure. Analogously to (1) and (2), the resulting matrix $\tilde{D}_{i, w}$ contains columns $\tilde{\mathbf{d}}_{m, i}^{(w)}$ that are defined as

\begin{tabular}{|c||c|c|c|c|c|}
\hline & $0-2 \mathrm{~Hz}$ & $2-18 \mathrm{~Hz}$ & $18-49 \mathrm{~Hz}$ & $49-51 \mathrm{~Hz}$ & $51-100 \mathrm{~Hz}$ \\
\hline \hline$\alpha$ & 0.1 & 0.1 & 0.4 & 0.2 & 0.4 \\
\hline$\beta$ & 1.1 & 1.4 & 1.1 & 1.2 & 1.1 \\
\hline$\gamma$ & 3 & 2 & 5 & 2.5 & 5 \\
\hline$\Theta$ & $\mathcal{P}_{95}$ & $\mathcal{P}_{100}$ & $\mathcal{P}_{80}$ & $\overline{\sigma_{m}}$ & $\mathcal{P}_{80}$ \\
\hline
\end{tabular}

Table 1. Set of parameters used for the automatic artifact reduction of EEG-data. Columns represent the band signals and rows the parameters used for the different sub-bands.

$$
\begin{aligned}
\tilde{\mathbf{d}}_{m, i}^{(w)}= & {\left[\tilde{d}_{m, i}^{(w)}[w(N-L)], \tilde{d}_{m, i}^{(w)}[w(N-L)+1], \ldots,\right.} \\
& \left.\tilde{d}_{m, i}^{(w)}[w(N-L)+N-1]\right]^{T} .
\end{aligned}
$$

From the entries $\tilde{d}_{m, i}^{(w)}[n]$ of the vector $\tilde{\mathbf{d}}_{m, i}^{(w)}$ the modified sub-band signal $\left\{\tilde{S}_{m, i}[n] \mid i=1,2, \ldots I\right\}$ is obtained by the overlap-add procedure

$$
\tilde{S}_{m, i}[n]=\sum_{w} \tilde{d}_{m, i}^{(w)}[n] .
$$

An appropriate synthesis filterbank corresponding to the analysis filterbank applied on $\tilde{S}_{m, i}[n]$ finally yields the resulting signal $\tilde{S}_{m}[n]$ of our artifact reduction method.

\subsection{Data}

Longterm EEG-data from six patients suffering from epilepsy were used with a total length of 46.5 days and containing 50 seizures. Data were recorded using 21 surface electrodes, applied according to the standard 10-20-system, with a sampling frequency $f_{s}=200 \mathrm{~Hz}$. Unequivocal seizure onsets (UEO) were determined by clinical experts.

\subsection{Parameters}

Parameters were chosen using a training data set consisting of five different windows lasting 15-30 seconds. This set was taken from one patient that was not included in the test data set used for the following calculations. Data containing a representative sample of different types of artifacts such as muscle activity, patient movement, loose electrodes or cable movement were visually selected. In addition, regions with seizure activity were included. A parameter set determined for these data is summarized in Tab.1. These obtained parameters were then used for artifact reduction on all other patients without modification.

Band limits of the filterbank were chosen such that different types of artifacts, line noise and seizure activity were separated into different sub-bands. This allows to identify not only the most dominant artifact in the EEG-signal, but also less apparent artifacts that dominate the variance within the respective band signal. Parameters for the adaptive threshold, i.e., $\alpha_{i}, \beta_{i}, \gamma_{i}$ and $\bar{\Theta}_{i, w}$, were chosen by visual comparison of the training data set before and after automatic artifact reduction. The whole parameter set was determined such that a good trade off between attenuating artifacts and maintaining the underlying activity was found. Smaller values and thus a stricter threshold were chosen for sub-bands, where strong artifacts were expected and more tolerant thresholds were used for sub-bands, which primarily contain activity of interest. 

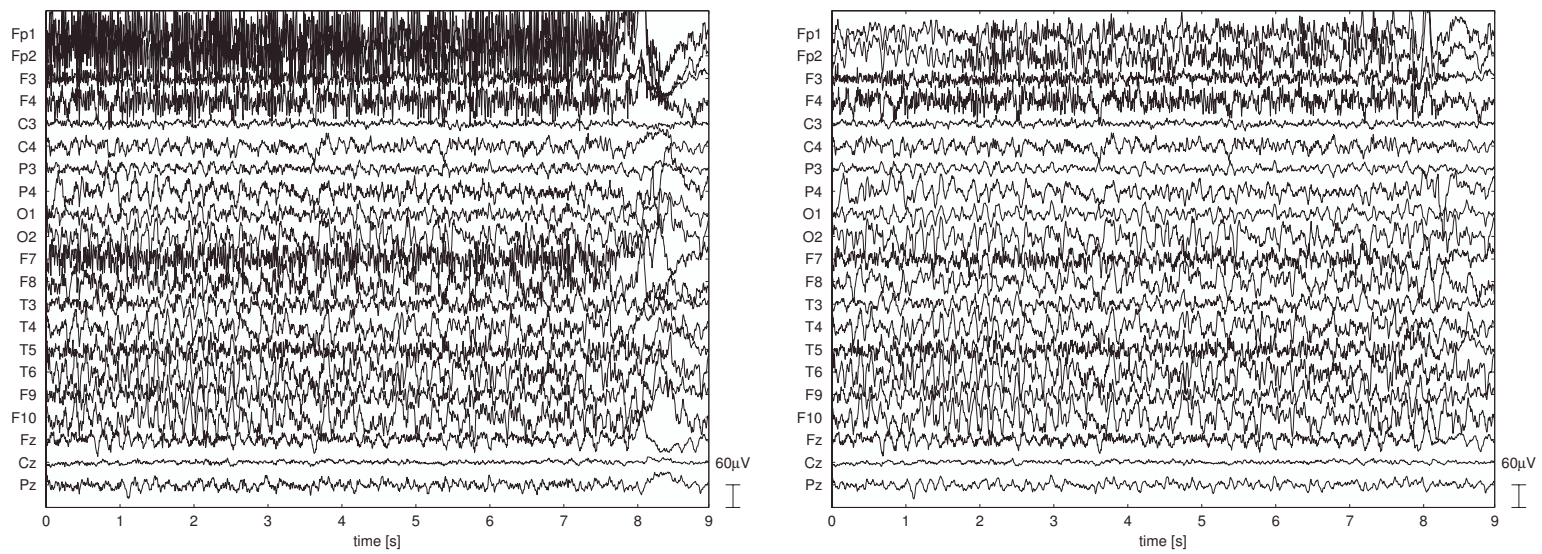

Fig. 1. left: the $y$-axis shows 21-channel EEG-data labeled with the electrode positions and the $x$-axis shows the time in seconds; data contain simultaneously high frequency muscle artifacts dominantly in the forehead region (FP1, FP2, F3, F4, F7), low frequency artifacts in FP1, Fp2, P4, F8, F10 and ictal patterns; right: after automatic artifact reduction: muscle artifacts are reduced significantly and low frequency artifacts vanished, while ictal patterns are preserved. as

For the window function $f[\nu]$, a tapered cosine function defined

$$
f[\nu]= \begin{cases}\frac{1+\cos \left(\pi+\frac{\nu}{L} \pi\right)}{2} & , \text { for } 0 \leq \nu<L \\ 1 & , \text { for } L \leq \nu<N-L \\ \frac{1+\cos \left(\frac{\nu-N+L}{L} \pi\right)}{2} & , \text { for } N-L \leq \nu<N\end{cases}
$$

was applied. The overlap $L$ and the overall window length $N$ was chosen corresponding to 0.5 seconds and two seconds, respectively.

In order to guarantee, that the threshold adapts within the first update steps of a recording, the initial value of $\Theta_{i, w}$ has to be chosen sufficiently high, although this leads to longer transient responses of the threshold in the beginning.

\section{RESULTS}

Fig.1 and Fig.2 show results of our automatic artifact reduction algorithm. The left part shows the raw EEG-recordings as a function of time, the right part shows the output of our algorithm. The parameters used for processing are summarized in Tab.1.

Fig.1 shows a typical example of high frequency artifacts during an epileptic seizure. Recordings on electrodes FP1 and FP2 are covered by permanent high frequency muscle activity during the first eight seconds. This activity is also present in recordings of electrodes F3, F4 and F7, but less dominant. In addition, high amplitude, low frequency activity, most likely caused by cable movement, is visible most apparently after eight seconds in FP1, FP2, P4, F8 and F10. Rhythmic seizure activity can be seen most pronounced in $\mathrm{O} 2$, F8, T4, T6 and F10. The right side of the figure shows the same data set after automatic artifact reduction. The results show that the high frequency artifacts are significantly reduced and EEG activity can now be observed in FP1 and FP2, which was first covered by the high frequency components. In addition, low frequency artifacts are also significantly reduced. In contrast, ictal activity throughout the example is clearly preserved.

Similar results are found for the EEG-recordings of a seizure free period shown in Fig.2. The layout is the same as in Fig.1. High am- plitude but very low frequency movement artifacts can be seen in almost all channels. They are most prominent from second three to five at recording site FP1, FP2, O2, F7, F8, T6, F9, F10 and Fz and from second nine to ten at Fp1, Fp2. The right side of this figure again shows the data segment after automatic artifact reduction. The low frequency disturbances are significantly reduced in all recording channels while the underlying EEG-activity was largely preserved.

Visual inspection of the whole data set of 46.5 days before and after automatic artifact reduction showed that almost all dominant artifacts were significantly reduced. In contrast, ictal activity was mostly unaffected by our methods. This was tested by visually comparing 30 seconds windows of raw EEG-data starting at seizure onset as defined by the UEO with the output of our algorithm. Ictal patterns remained unaltered in 48 out of 50 seizures $(96 \%)$. In the other two seizures, both recorded from the same patient, rhythmic activity was attenuated in the beginning of the seizure.

\section{DISCUSSION}

In this study we proposed a novel algorithm for the reduction of artifacts in EEG-data. Our results demonstrate that our method effectively attenuates different types of artifacts in EEG-signals caused by, e.g., muscle contractions, body motion, eye blinks, loose electrodes or cable movement, and that no manual interaction is required. Furthermore, only a small training data set is sufficient for an effective reduction.

This new approach strongly enhances automatic signal processing. Features, e.g., used for the detection or prediction of epileptic seizures (for an overview see, e.g., [11]) often mistakenly detect seizure activity in regions, where high amplitude artifact activity is found. Application of our automatic artifact reduction can significantly improve the performance of these methods. A further study will quantify the performance gain of seizure detection algorithms after our automatic artifact reduction. In clinical practise the original data should still be considered for visual inspection of EEG-signals.

Our results show that equal parameters can be used for many pa- 

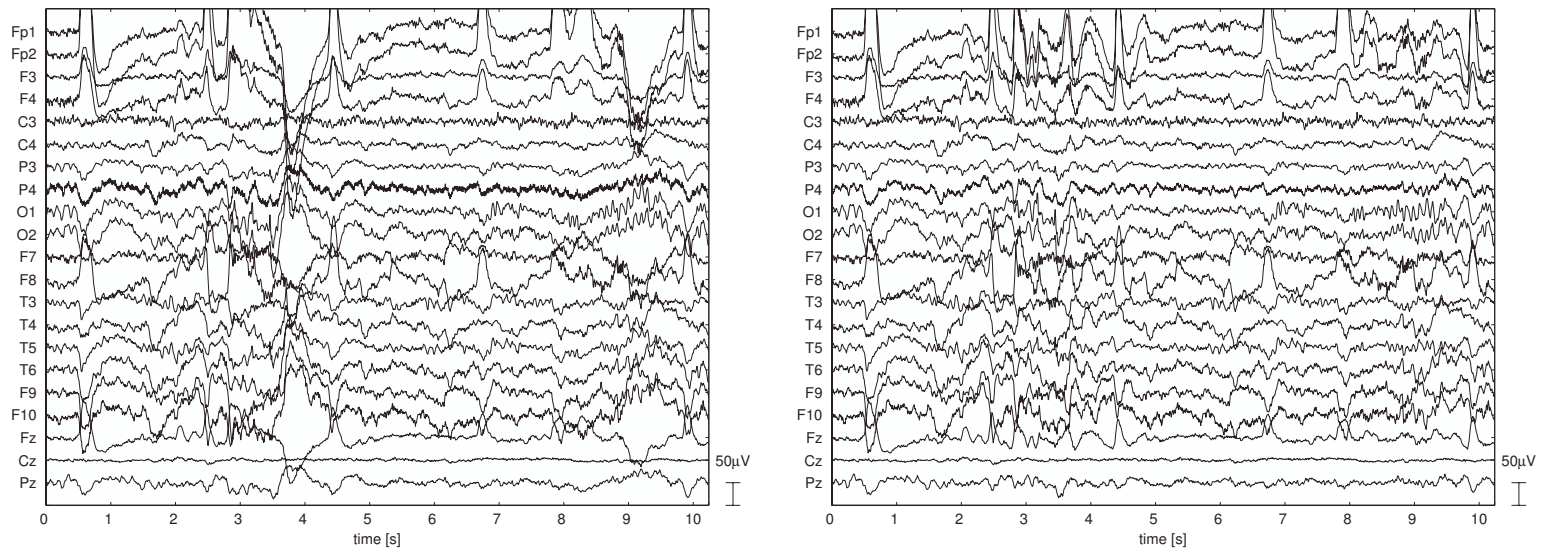

Fig. 2. For layout see Fig.1 left: EEG-data containing low frequency artifacts. right: Data segment after automatic artifact reduction. The low frequent artifacts are significantly reduced.

tients. This is indicated by the fact that we were using the same parameter set based on training data from one patient for the whole test data set of 46.5 days recorded from five different patients. This strongly suggests that a universal parameter set for a given type of recording can be used. Such universal parameter sets are particularly important in online applications and will enhance the range of application of our approach. For a further performance gain, different parameter sets for different types of epilepsy might be useful.

For the proposed method, automatic parameter optimization could be performed. As an optimization criterion the output of a seizure detection algorithm for a training data set can be used. An optimal parameter set should yield a good trade off between few false positive and few false negative seizure detections. Such automatic parameter identification can further increase the performance of our artifact reduction method.

\section{CONCLUSION}

In this paper we proposed a novel method for the automatic reduction of artifacts in EEG-data. The results confirm that our method is able to significantly reduce different types of artifacts like, e.g. high frequency muscle artifacts or low frequency artifacts caused by the movement of patients. Visual inspection of data showed that the approach is a very efficient method to eliminate artifacts and significantly improves the quality and usefulness of recorded data. The results further indicate that it is not necessary to identify the parameters for every patient individually. In practice this will be particularly important because no training period is required.

\section{REFERENCES}

[1] A. Delorme, T. Sejnowski, and S. Makeig, "Enhanced detection of artifacts in EEG data using higher-order statistics and independent component analysis," Neuro Image, vol. 34, 2007.

[2] A. Delorme, S. Makeig, and T. Sejnowski, "Automatic artifact rejection for EEG data using high-order statistics and independent component analysis," International Workshop on ICA, 2001.
[3] G. Barbati, C. Porcaro, F. Zappasodi, P.M. Rossini, and F. Tecchio, "Optimization of an independent component analysis approach for artifact identification and removal in magnetoencephalographic signals," Clinical Neurophysiology, vol. 115, 2004.

[4] H. Nam, T.G. Yim, J.B. Oh, and S.K. Lee, "Independent component analysis of ictal EEG in medial temporal lobe epilepsy," Epilepsia, vol. 43, 2002.

[5] E. Urrestarazu, J. Iriartre, M. Alegre, M. Valencia, C. Viteri, and J. Artieda, "Independent component analysis removing artifacts in ictal recordings," Epilepsia, vol. 45, 2004.

[6] C. J. James and O. J. Gibson, "Temporally constrained ICA An application to artifact rejection in electromagnetic brain signal analysis," IEEE Transactions on Biomedical Engeneering, vol. 50, 2003.

[7] S. Park, H. Lee, and S. Choi, "ICA+OPCA for artifact-robust classification of EEG data," IEEE XIII Workshop on Neural Networks for Signal Processing, 2003.

[8] A. Flexer, H. Bauer, J. Pripfl, and G. Dorffner, "Using ICA for removal of ocular artifacts in eeg recorded from blind subjects," Neural Networks, vol. 18, 2005.

[9] W. Lu and J.C. Rajapakse, "Approach and applications of constrained ICA," IEEE Tranactions on Neural Networks, vol. 16, 2005.

[10] P. LeVan, E. Urrestarazu, and J. Gotman, "A system for automatic artifact removal in ictal scalp EEG based on independent component analysis and bayesian classification," Clinical Neurophysiology, vol. 117, 2006.

[11] F. Mormann, Thomas Kreuz, Christoph Rieke, Ralph G. Andrzejak, Alexander Kraskov, Peter David, Christian E. Elger, and Klaus Lehnertz, "On the predictability of epileptic seizures," Clinical Neurophysiology, vol. 116, 2005. 